\title{
Introduction to IS Risk and Decision-Making Minitrack
}

\author{
Dan Port \\ University of Hawaii at Manoa \\ dport@hawaii.edu
}

\author{
Joel Wilf \\ Jet Propulsion Laboratory \\ jwilf@jpl.nasa.gov
}

\section{Introduction}

The Information System (IS) today is a vital and ever-present part of our lives, from our computers and mobile devices to our online business and social sites; from the systems that are embedded in our planes, cars, and appliances to the medical devices that may be embedded in our own bodies. They are a source of benefits, but also of risk. Everyone who is responsible for building, acquiring, or maintaining these systems face questions of risk during the IS life cycle. Are the systems safe? Are they reliable? Are they secure? Based on answers to these questions, decisions must be made: should the new software be released or held for more testing? Should the next rocket to Mars be launched? Should a new insulin pump be approved for human use?

This minitrack had contributions from researchers and practitioners, in both academia and industry, who provide insight into decision-making throughout the IS life cycle and its impact on IS risk. Addressing questions such:

What methods are being used for risk-informed decision-making?

How are trade-offs for risk vs. cost, schedule, and performance handled?

How are decisions affected by software or systems assurance?

How are decisions affected by the cognitive biases of the decision-makers and the culture of the IS development organization?

Submissions were cross-disciplinary, including social and psychological factors in addition to technical ones.
Topics included but were not restricted to:

- Methods for risk-informed decision making

- Risk analyses for critical decisions, such as "certification for launch readiness"

- Handling trade-offs for risk, cost, schedule, and performance

- Assuring safety, reliability, or security during the IS life-cycle

- The role of government and industry standards

- The effect of cognitive biases on risk perception and decision-making

- The effect of organizational culture or economic pressures on risk perception and decision-making

- Analyses of actual success or failure of risk critical decisions

- $\quad$ Processes and tools (e.g., risk analysis methods or decision support systems) with the potential for improving risk-critical decision outcomes 